\title{
Retraction Note to: A hybrid SVM-FFA method for prediction of monthly mean global solar radiation
}

\author{
Shahaboddin Shamshirband ${ }^{1} \cdot$ Kasra Mohammadi $^{2} \cdot$ Chong Wen Tong $^{3} \cdot$ Mazdak Zamani $^{4} \cdot$ Shervin $^{\text {Motamedi }}{ }^{5} \cdot$ \\ Sudheer $\mathrm{Ch}^{6}$
}

Published online: 9 March 2020

(C) Springer-Verlag GmbH Austria, part of Springer Nature 2020

Retraction Note to: Theor Appl Climatol (2016) 125:53-65 https://doi.org//10.1007/s00704-0151482-2

The Editor-in-Chief has retracted this article [1] because validity of the content of this article cannot be verified. This article showed evidence of peer review and authorship manipulation. Shahaboddin Shamshirband disagrees with this re- traction. Authors Abdullah Kasra Mohammadi, Chong Wen Tong, Mazdak Zamani, Shervin Motamedi, and Sudheer Ch have not responded to correspondence about this retraction.

[1] Shamshirband, S., Mohammadi, K., Tong, C.W. et al. Theor Appl Climatol (2016) 125: 53. https://doi.org/10. 1007/s00704-015-1482-2

The online version of the original article can be found at https://doi.org/ $10.1007 / \mathrm{s} 00704-015-1482-2$

Shahaboddin Shamshirband

shamshirband@um.edu.my

Kasra Mohammadi

kasra278@yahoo.com

1 Department of Computer System \& Technology, Faculty of Computer Science \& Information Technology, University of Malaya, Kuala Lumpur, Malaysia

2 Faculty of Mechanical Engineering, University of Kashan, Kashan, Iran

3 Department of Mechanical Engineering, Faculty of Engineering, University of Malaya, 50603 Kuala Lumpur, Malaysia

4 Advanced Informatics School, Universiti Teknologi Malaysia, 54100 Kuala Lumpur, Malaysia

5 Institute of Ocean and Earth Sciences (IOES), University of Malaya, 50603 Kuala Lumpur, Malaysia

6 Department of Civil and Environmental Engineering, ITM University, Gurgaon, Haryana 122017, India 